\title{
ENTROPY FOR SMOOTH ABELIAN ACTIONS
}

\author{
DAVID FRIED
}

\begin{abstract}
We gencralize the usual notions of metric and topological entropy for flows to actions of compactly generated abelian Lic groups. Unlike previous generalizations, ours is nontrivial for smooth actions of $\mathbf{R}^{\prime \prime} . n \geqslant 2$. We prove some elementary properties of our definitions and we relate them to characteristic exponents and the entropy conjecture.
\end{abstract}

The essential numerical invariant in the ergodic theory of a single transformation is the metric entropy defined by Kolmogorov and Sinai. An analogous definition for measure preserving actions of $\mathbf{Z}^{2}$ (and certain other abelian groups) was given by various authors $[\mathbf{K}, \mathbf{C}, \mathbf{K W}, \mathbf{T}]$. In a different setting, the topological entropy of a continuous map on a metric space was defined and generalized to $\mathbf{Z}^{2}$ (et al.) actions [AKM, R]. These definitions have the undesirable property that the entropy of a smooth $\mathbf{Z}^{2}$ action is always zero $[\mathbf{C}, \mathbf{R}]$. In this note we will give different definitions of the metric and topological entropies of an action of $\mathbf{Z}^{2}$ (et al.) that do not degenerate in the smooth case. We will establish their elementary properties and relate them to characteristic exponents and the entropy conjecture.

We thank Don Ornstein for helpful discussions.

1. Metric entropy of a $G$ action. Throughout this paper $G$ will denote a compactly generated abelian Lie group with a fixed Haar measure. We need several facts about this category of groups. Every $G$ is a finite product of lines, circles, and cyclic groups. $G$ has a unique maximal compact subgroup $K$. The space $(G / K) \otimes \mathbf{R}$ is a finite dimensional real vector space $V=V(G)$. We will denote the image of $g \in G$ in $V$ by $g \otimes 1$. We give $G \otimes 1 \cong G / K \subset V$ the quotient Haar measure and we give $V$ the Haar measure $d$ vol such that the torus $V /(G \otimes 1)$ has quotient Haar measure of total mass 1 . In the important case when $G=\mathbf{R}^{m} \otimes \mathbf{Z}^{n}$ with the usual Haar measure, $V$ is just $\mathbf{R}^{m+n}$ with the usual volume form. When $G$ is compact, $V$ is 0 with the same total mass, $\operatorname{mass}(V)=\operatorname{mass}(G)$.

Let $(X, \mu)$ be a probability space with a measurable $G$ action by measure preserving transformations $T_{g}, g \in G$. We canonically define a new probability space $\left(X^{\prime}, \mu^{\prime}\right)$ with a measurable $V$ action by measure preserving transformations $T_{v}^{\prime}$, $v \in V$, as follows. Let $\sim$ be the equivalence relation on $X \times V:(x, v) \sim\left(T_{g}(x), v-\right.$ $g \otimes 1)$, for all $x \in X, v \in V, g \in G$. Let $X^{\prime}=X \times V / \sim$ and let $T_{v}^{\prime}\left(x_{0}, v_{0}\right)=$ $\left(x_{0}, v_{0}+v\right)$. Observe that $X^{\prime}$ is a bundle over a torus $B=V /(G \otimes 1)$ with fiber

Received by the editors August 31, 1981 and, in revised form, May 7, 1982

1980 Mathematics Subject Classification. Primary 28D20: Secondary 54H20, 57S20, 58F11.

'Partially supported by NSF Grant \# MCS 8003622. 
$F=X / K$ the factor space of the action of $K$ on $X$. We give $F$ the factor measure induced from $\mu$ and $B$ the Haar probability measure and let $\mu^{\prime}$ be the (local) product measure on $X^{\prime}$. This construction generalizes the "flow under a function" construction of ergodic theory for return time one when $G=\mathbf{Z}$ and $V=\mathbf{R}$.

Now consider the metric entropy as a function on $V, f(v)=h_{\mu}\left(T_{v}^{\prime}\right), v \in V$. Clearly $F: V \rightarrow[0, \infty]$ is measurable. Let $U=\{v \in V \mid f(v) \leqslant 1\}$ be the measurable "unit ball" for $f$ (in the examples of $\S 2, f$ is actually a seminorm on $V$ ). Let $\nu$ be the volume of the generalized octahedron $\theta=\left\{\left(x_{i}\right) \in \mathbf{R}^{d}|\Sigma| x_{i} \mid<1\right\}$, where $d=\operatorname{dim} V$. Our generalization of metric entropy is

Definition. $h_{\mu}^{*}(G, X)=\nu /$ vol $U$.

REMARKS. (1) If $G=\mathbf{R}$ or $\mathbf{Z}$ with the usual measure then $\theta=[-1,+1]$ and $U=\left\{v \in \mathbf{R}|| v \mid \leqslant 1 / h_{\mu}\left(T_{1}\right)\right\}$. Thus $h_{\mu}^{*}(G, X)=h_{\mu}\left(T_{1}\right)$ generalizes the metric entropy of a flow or invertible transformation.

(2) If $V=0\left(\Leftrightarrow G\right.$ is compact) then $h_{\mu}^{*}(G, X)=1 / \operatorname{mass}(G)$.

(3) Suppose $G=\mathbf{R}^{n}$ and $h_{\mu}\left(T_{g}\right)<\infty$ for all $g \in G$. By Abramov's formula (cited below) $U$ contains a nontrivial segment in each direction so that vol $U>0$. Hence when each $T_{g}$ has finite entropy, the action as a whole has finite entropy.

(4) Suppose $G$ acts on a factor space $(\bar{X}, \bar{\mu})$ of $(X, \mu)$. Then $h_{\bar{\mu}^{\prime}}\left(\bar{T}_{v}^{\prime}\right) \leqslant h_{\mu^{\prime}}\left(T_{v}^{\prime}\right)$ for all $g$ and it follows that $h_{\bar{\mu}}^{*}(G, \bar{X}) \leqslant h_{\mu}^{*}(G, X)$. This also holds if $\bar{X}$ is an invariant subspace of $X$ with $\mu(\bar{X})>0$ and $\bar{\mu}(A)=\mu(A) / \mu(\bar{X})$ is the induced probability measure.

(5) We can easily extend this definition to noninvertible transformations, semiflows and actions of a semigroup $S=\left(\mathbf{R}^{+}\right)^{a} \oplus \mathbf{R}^{b} \oplus\left(\mathbf{Z}^{+}\right)^{c} \oplus \mathbf{Z}^{d}$. We use an inverse limit construction to produce a new probability space $(\bar{X}, \bar{\mu})$ on which $S$ acts by invertible transformations. Then we let $h_{\mu}^{*}(S, X)=h_{\bar{\mu}}^{*}\left(\mathbf{R}^{a+b} \oplus \mathbf{Z}^{c+d}, \bar{X}\right)$.

We now prove two properties of the invariant $h_{\mu}^{*}$. The first concerns product actions. Let $G_{i}$ act on $\left(X_{i}, \mu_{i}\right)$ as above, $i=1,2$. Give $G=G_{1} \oplus G_{2}$ the product Haar measure. Let $X=\left(X_{1} \times X_{2}, \mu_{1} \times \mu_{2}\right)$ with the product $G$ action. With the convention $0 \cdot \infty=\infty$, we have

Proposition 1. $h_{\mu}^{*}(G, X)=h_{\mu_{1}}^{*}\left(G_{1}, X_{1}\right) h_{\mu_{2}}^{*}\left(G_{2}, X_{2}\right)$.

Proof. Since $V(G)$ is additive, the vector spaces $V_{1}, V_{2}$ and $V$ satisfy $V=V_{1} \oplus V_{2}$. For $v=\left(v_{1}, v_{2}\right) \in V$, we have $h_{\mu^{\prime}}\left(T_{v^{\prime}}^{\prime}\right)=h_{\mu_{1}}\left(T_{v_{1}}^{\prime}\right)+h_{\mu_{2}^{\prime}}\left(T_{v_{2}}^{\prime}\right)$. In other words, the functions $f_{i}: V_{i} \rightarrow[0, \infty]$ and $f: V \rightarrow[0, \infty]$ satisfy $f\left(v_{1}, v_{2}\right)=f_{1}\left(v_{1}\right)+f_{2}\left(v_{2}\right)$. As $f_{1}$ and $f_{2}$ are positively homogeneous the "unit balls" $U_{i} \subset V_{i}$, and $U \subset V$ satisfy $\operatorname{vol} U=\operatorname{vol}_{1} U_{1} \cdot \operatorname{vol}_{2} U_{2} \cdot C$, where $C$ is a dimensional constant. The octahedra $\theta_{1}$, $\Theta_{2}$ and $\mathcal{O}$ satisfy a corresponding equation and we divide and cancel the $C$ 's. Q.E.D.

REMARK. If $G_{2}$ is trivial this proposition gives the dependence of $h_{\mu}^{*}$ on the choice of Haar measure on $G$.

We now consider a co-compact subgroup $G^{\prime} \subset G$. There is a natural Haar measure on $G^{\prime}$ such that if $G / G^{\prime}$ is given the quotient Haar measure and $\Gamma$ is a discrete co-compact subgroup of $G^{\prime}$ we have $\operatorname{mass}(G / \Gamma)=\operatorname{mass}\left(G / G^{\prime}\right) \operatorname{mass}\left(G^{\prime} / \Gamma\right)$. When $G^{\prime}$ is discrete this is just the counting measure on $G^{\prime}$. The entropy of the 
action of $G^{\prime}$ on $X$ is given by the following generalization of Abramov's formula $h_{\mu}\left(\varphi_{t}\right)=|t| h_{\mu}\left(\varphi_{1}\right)$ for the cases $G=\mathbf{R}, G^{\prime}=t \mathbf{Z}$.

Proposition 2. $h_{\mu}^{*}\left(G^{\prime}, X\right)=\operatorname{mass}\left(G / G^{\prime}\right) h_{\mu}^{*}(G, X)$.

Proof. The natural map $G^{\prime} \otimes \mathbf{R} \rightarrow G \otimes \mathbf{R}$ is an isomorphism of vector spaces that increases volumes by a factor of $\operatorname{mass}\left(G / G^{\prime}\right)$. As the dimensional constant $\nu$ in the definition of $h_{\mu}^{*}$ is the same for $G^{\prime}$ and $G$, we need only show the functions $f, f^{\prime}$ : $V \rightarrow[0, \infty]$ are equal. This depends on Abramov's formula. By passing to the action of $G / K$ on $F=X / K$, we may suppose $K=0$. By induction, it suffices to prove the proposition when $G / G^{\prime}$ is a circle or a finite cyclic group.

Suppose it is a circle. For some $H \subset G^{\prime}$ we have $G^{\prime}=H \oplus \mathbf{Z}, G=H \oplus \mathbf{R}$. Letting $W=V(H)$ we have $V=W \oplus \mathbf{R}$. Let $\left(V, X^{\prime}\right)$ be the suspension of the action $(G, X)$ as above and let $\psi_{t}$ be the flow on $X^{\prime}$ corresponding to the factor $\mathbf{R} \subset V$. The suspension of $\left(G^{\prime}, X\right)$ is $X^{\prime \prime}=X^{\prime} \times \mathbf{R} /\left(x^{\prime}, r\right) \sim\left(\psi_{n} x^{\prime}, r-n\right), n \in \mathbf{Z} . V$ acts on $X^{\prime \prime}$ by $T_{v}^{\prime \prime}\left(x^{\prime}, r\right)=\left(T_{w}^{\prime} x^{\prime}, r+t\right)$ for $v=(w, t)$. We must show that $T_{v}^{\prime}$ and $T_{v}^{\prime \prime}$ have the same entropy for all $v \in V$.

By the Abramov formula, it is sufficient to show this when $v=(w, n), n \in \mathbf{Z}$. Then $T_{n}^{\prime \prime}\left(x^{\prime}, r\right) \approx\left(\psi_{n} T_{w}^{\prime} x^{\prime}, r\right)=\left(T_{v}^{\prime} x^{\prime}, r\right)$. So $T_{v}^{\prime \prime}$ is just a parametrized family of $\left(T_{v}^{\prime}\right)^{\prime}$ 's and they have equal entropy.

Finally suppose $G / G^{\prime}$ is cyclic of order $n$. For some $H$ we have $G=H \oplus \mathbf{Z}$, $G^{\prime}=H \oplus n \mathbf{Z}$. By considering $G^{*}=H \oplus \mathbf{R}$ as well, we reduce to the preceding case. Q.E.D.

2. Smooth actions preserving an absolutely continuous measure. We now suppose that $X$ is a compact $C^{\infty}$ manifold without boundary, that $\mu$ is absolutely continuous with respect to Lebesgue measure and that the transformations $T_{g}, g \in G$, are diffeomorphisms of class $C^{1+\varepsilon}$, some $\varepsilon>0$. Using a well-known result of Kushnirenko, we have $h_{\mu}\left(T_{g}\right)<\infty$ for all $g$. Using results of Pesin, we show

Proposition 3. $h_{\mu}\left(T_{x+y}\right) \leqslant h_{\mu}\left(T_{x}\right)+h_{\mu}\left(T_{y}\right)$ for all $x, y \in G$.

Proof. Since $h_{\mu}(T)$ is an affine function of the invariant measure $\mu$, we may suppose that $\mu$ is $G$-ergodic.

Fix $g$. By Oseledec's ergodic theorem [O], there is almost everywhere a $T_{g}$-invariant splitting of the tangent bundle such that the growth of vectors in each subspace under the iterated differential is constant. As $G$ is abelian this splitting is preserved by $G$ and the splittings corresponding to elements $g_{1}, g_{2} \in G$ are compatible. So for each $g \in G$ and $p \in X$ we have $X_{p}=W_{1} \oplus \cdots \oplus W_{m}$ and characteristic exponents $\mu_{i} \in \mathbf{R}, i=1, \ldots, m$, so that $v \in W_{i}, v \neq 0$, satisfies $\lim \frac{1}{n} \log \left\|D T_{g}^{n} v\right\|=$ $\mu_{i}$. By the ergodicity of $\mu$, the value of $\mu_{i}$ is independent of $p$ and depends only on $g$. One also has $\mu_{i}\left(g_{1}\right)+\mu_{i}\left(g_{2}\right)=\mu_{i}\left(g_{1}+g_{2}\right)$, for $g_{1}, g_{2} \in G$.

Pesin proved [P] that $h_{\mu}\left(T_{g}\right)=\int \chi_{+}(g) d \mu$ where $\chi_{+}(g)=\Sigma_{i} \mu_{i}(g) \operatorname{dim} W_{i}$, over all $i$ for which $\mu_{i}(g)>0$. As $\chi_{+}(g)$ is constant, we have $h_{\mu}\left(T_{g}\right)=\sum \mu_{i}(g) \operatorname{dim} W_{i}=$ $\Sigma_{i}\left(\operatorname{dim} W_{i}\right) \max \left(0, \mu_{i}(g)\right)$. The subadditivity property is now obvious. Q.E.D.

REMARKS. This proposition was known to Ornstein (personal communication) who has examples of measure preserving $\mathbf{Z}^{2}$ actions for which entropy is not 
subadditive. Proposition 3 says such actions cannot be smoothed. Thouvenot independently has such examples as well.

COROllary. If $(G, X)$ is ergodic and smooth (as above) then the unit ball $U$ of $\$ 1$ is a convex polyhedron. $h_{\mu}^{*}(G, X)$ is finitely computable from the characteristic exponents.

Proof. By Proposition 2 it suffices to show this when $G=\mathbf{Z}^{a}$. Then $V$ acts smoothly on $Y^{\prime}$ and we apply Proposition 3 to $V$.

From the proof we have $h_{\mu}\left(T_{v}^{\prime}\right)=\sum_{i=1}^{m}\left(\operatorname{dim} W_{i}\right) \max \left(0, \mu_{i}(n)\right)$. For each $S \subset$ $\{1, \ldots, m\}$ let $\pi_{S}(v)=\Sigma_{i \in S}\left(\operatorname{dim} W_{i}\right) \mu_{i}(v)$. Then $f(v)=\sup _{S} \pi_{S}(v)$ is a seminorm, $U$ is a convex polyhedron, and the faces of $U$ are determined by the characteristic exponent functionals $\mu_{i}: \mathbf{Z}^{a} \rightarrow \mathbf{R}$. Q.E.D.

3. Topological entropy of $G$-actions. In this section $Y$ denotes a metric space with a given continuous $G$ action. The topological entropy $h\left(T_{g}\right)[\mathbf{A K M}, \mathbf{S}]$ of these transformations will be used to define an entropy invariant for this action. With an eye to certain applications, we proceed a little differently than in $\$ 1$.

Let $\widetilde{y}(V)=\{F: G \rightarrow[0, \infty]|F(x+y) \leqslant F(x)+F(y), F(t x)=| t \mid F(x)$, for $x, y$ $\in V, t \in \mathbf{R}\}$ be the family of all extended real valued seminorms on $V$. Let $\|\cdot\| \in \widetilde{F}(V)$ be the maximal element satisfying

$$
\|g \otimes 1\| \leqslant h\left(T_{g}\right), \quad \text { all } g \in G .
$$

Let $U^{c}$ be the unit ball for $\|\cdot\|$.

Definition. $h^{c}(G, X)=\nu / \operatorname{vol}\left(U^{c}\right)$.

The superscript $c$ is a reminder that the unit ball in $V$ has been chosen convex.

All the remarks of $\$ 1$ and Proposition 1 hold for $h^{c}$ with the obvious changes. For Proposition 2 one must assume that $h\left(T_{g}\right)$ is a continuous function of $g \in G$. This hypothesis is automatic if $G$ is discrete and it is not hard to prove that it holds when $\operatorname{dim}(G / K) \leqslant 1$. This raises the questions

Question 1. If $f$ and $g$ are commuting homeomorphisms of a metric space, is $h(f \circ g) \leqslant h(f)+h(g)$ ?

Question 2. If $G$ acts continuously on $Y$ (or smoothly on a manifold $M$ ) is $h\left(T_{g}\right.$ ) continuous in $G$ ?

If Question 1 holds then Question 2 follows since convex functions are continuous. But there may be counterexamples to Question 1 along the lines of the Ornstein/Thouvenot examples.

In the special case when $Y=E$ is Euclidean space and the action of $G$ is linear, the answer to Question 1 is yes. This is so because the entropy of the linear transformation $L$ is $h(L)=\sum n_{i} \log \left|\lambda_{i}\right|$ where $\lambda_{i}$ runs over the eigenvalues of $L$ of modules $>1$ and $n_{i}$ is the corresponding multiplicity. The transformations $T_{g}$ can be simultaneously upper triangulated over $\mathbf{C}$, since $G$ is abelian. We find that $h\left(T_{g}\right)$ is subadditive and (as in $\S 2)$ the unit ball is a polyhedron and $h^{*}(G, E)$ is computable.

Following Shub, we say a continuous map $f$ of a compact manifold satisfies the entropy conjecture if $h(f)$ is at least as large as the logarithm of the spectral radius of 
the action of $f$ on homology. Our definition $h^{c}(G, Y)$ fits well into this setting. For instance

Proposition 4. Let $Y=T^{n}$ with some continuous $G$ action. Let $E=H_{1}(Y ; \mathbf{R})$ with the induced $G$ action. Then $h^{\prime}(G, Y) \geqslant h^{c}(G, E)$.

Proof. Misiurewicz and Przytycki proved that $h\left(T_{g}\right) \geqslant h\left(T_{g^{*}}\right)$ for $T_{g^{*}}: H_{1}\left(T^{n}, \mathbf{R}\right)$ [MP, B]. It follows that the seminorm \|\|$_{Y}$ for the action of $G$ on $Y$ dominates \|\|$_{E}$, so that the unit balls satisfy $U_{Y} \subset U_{E}$. Thus $\operatorname{vol} U_{Y} \leqslant \operatorname{vol} U_{E}$ and $h^{c}(G, E) \leqslant$ $h^{c}(G, Y)$. Q.E.D.

Now let $Y$ be a connected finite complex and define $F_{1}: G \rightarrow[0, \infty)$ to be the growth rate of the maps induced on the fundamental group of $Y$,

$$
F_{1}(g)=\gamma\left(\left(T_{g}\right)_{*}: \pi_{1} Y \leftrightarrow\right)
$$

$[\mathbf{B}, \mathbf{S}]$. One can easily check that endomorphisms of a finitely generated group satisfy $\gamma\left(f^{n}\right)=|n| \gamma(f)$ and $\gamma\left(f_{1} \circ f_{2}\right) \leqslant \gamma\left(f_{1}\right)+\gamma\left(f_{2}\right)$ when $f_{1} \circ f_{2}=f_{2} \circ f_{1}$. Thus $F$ is a seminorm on $G$. Let $U_{1} \subset V$ be the associated unit ball. Using the known result $h\left(T_{g}\right) \geqslant \gamma\left(\left(T_{g}\right)_{*}\right)[\mathbf{B}, \mathbf{S}]$ we see as in the preceding proof,

Proposition 5. $h^{c}(G, Y) \geqslant \nu / \operatorname{vol} U_{1}$.

Likewise if the entropy conjecture holds for each transformation $T_{g}: Y \rightarrow Y$ on the $i$ dimensional homology $H_{i}(Y ; \mathbf{R}), i>1$, we let $F_{i}: G \rightarrow[0, \infty)$ assign to $g \in G$ the logarithm of the spectral radius of the map $\left(T_{g}\right)_{*}: H_{i}(Y, \mathbf{R}) \hookleftarrow$. Then since $G$ is abelian, $F_{i}$ is a seminorm. By assumption, $F_{i}$ is dominated by $h\left(T_{g}\right)$. So for the unit ball $U_{l}$ determined by $F_{i}$, we have $h^{c}(G, Y) \geqslant \nu / \operatorname{vol} U_{l}$. For instance

Proposition 6. Let $Y$ be a compact affine manifold with a $G$ action that preserves the affine structure. Then for all $i, h^{c}(G, Y) \geqslant \nu / \mathrm{vol} U_{i}$.

Proof. This follows from the preceding discussion since the entropy conjecture is known for affine maps [FS]. Q.E.D.

We conclude by comparing the definitions of $\S 1$ and this section for the systems of §2. Using Goodwyn's Theorem that the metric entropy does not exceed topological entropy, we see that the seminorm $h_{\mu}^{*}\left(T_{g}\right)$ of $\$ 2$ is a lower bound for the seminorm of this section. Thus $h_{\mu}^{*}(G, X) \leqslant h^{c}(G, X)$.

\section{REFERENCES}

[AKM] R. L. Adler, A. G. Konheim and M. H. McAndrew, Topological entropy. Trans. Amer. Math. Soc. 114 (1965), 309-319.

[B] R. Bowen, Entropy and the fundamental group, Lecture Notes in Math., vol 668, Springer-Verlag. New York, 1978.

[C] J. P. Conze, Entropie d'un groupe abelian de transformations, Z. Wahrsch. Verw. Gebiete 25 (1972), $11-30$.

[FS] D. Fried and M. Shub, Entropy, linearity and chain recurrence. Inst. Hautes Etudes Sci. Publ. Math. 50 (1980), 203-214.

[KW] Y. Katznelson and B. Weiss, Commuting measure preserving transformations, Israel J. Math. 12 (1972), 161-173.

[K] A. A. Kirillov, Dynamical systems, factors and group representations, Russian Math Surveys 22 (1967). 
[MP] M. Misiurewicz and F. Przytycki, The entropy conjecture on tori, preprint.

[O] V. I. Oseledec, A multiplicative ergodic theorem. Lyapunov characteristic numbers for dynamical systems. Trans. Moscow Math. Soc. 19 (1968), 197-231.

[P] J. B. Pesin, Lyapunov characteristic exponents and smooth ergodic theory, Russian Math. Surveys 32 (1977), 55-114.

[R] D. Ruelle, Thermodynamic formalism, Addison-Wesley, Reading, Mass., 1978.

[S] M. Shub, Some dynamics of pseudo-Anosov diffeomorphisms, Astérisque 66-67 (1979), 181-208.

[T] J. P. Thouvenot, Convergence en movenne de l'information pour l'action de $\mathrm{Z}^{2}, \mathrm{Z}$. Wahrsch. Verw. (jebiete 24 (1972), 135-137.

Department of Mathematics, University of California at Santa Cruz, Santa Cruz, CaliFORNIA 95064

Current address: Department of Mathematics, IHES, 91440 Bures sur Yvette, France 